\title{
Luiz Beltrão, Décio Pignatari, Muniz Sodré y Sérgio Capparelli. Aportes teóricos brasileños al pensamiento latinoamericano de la
} comunicación

\section{Luiz Beltrão, Décio Pignatari, Muniz Sodré and Sérgio Capparelli. Brazilian Theoretical Contributions to Latin American Thought in Communication}

\author{
Gustavo A. León Duarte ${ }^{(*)}$ \\ Universidad de Sonora - México \\ gustavoadolfoleon@gmail.com
}

\section{Resumen}

El documento centra su atención en definir cuáles son y en qué consisten algunas características teóricas de hondo arraigo que identifican a cuatro investigadores emblemáticos de los estudios de la Comunicación en Brasil: Luiz Beltrão, Décio Pignatari, Muniz Sodré y Sérgio Capparelli. El texto trata de definir por qué éstos aportes teóricos brasileños son trascendentales para establecer un pensamiento comunicacional propio en los estudios de la comunicación en Brasil y en América Latina en general. El colofón identitario de los especialistas brasileños aquí analizados parece

\begin{abstract}
The paper centers on defining some structural characteristics that identify four emblematic researchers of the Sciences of the Communication in Brazil: Luiz Beltrão, Décio Pignatari, Muniz Sodré and Sérgio Capparelli. The paper attempts to define why these Brazilian theoretical contributions to establish a communication thought in itself in communication studies in Brazil and Latin America in general. The closure that identifies the Brazilian specialists analyzed here is the ethical-political platform of communication, amalgamated with a multidisciplinary approach, and a
\end{abstract}


«Luiz Beltrão, Décio Pignatari, Muniz Sodré y Sérgio Capparelli. Aportes teóricos...»

no ser otro que la plataforma éticopolítica de la comunicación, amalgamada con una visión multidisciplinar, crítica y socio-histórica de sus temas, objetos y métodos de estudio.

Palabras clave: investigación de la comunicación, ética, epistemología, Latinoamérica. socio-historical criticism of its subjects, objects and methods of study.

Keywords: communication research, ethics, epistemology, Latin America. 


\section{Presentación}

En este texto se pretende ofrecer una visión general sobre cuáles son algunos de los principales aportes teóricos brasileños al Pensamiento Latinoamericano de la Comunicación (PLC). Particularmente, me concentro en definir las características teóricas que considero como de hondo arraigo en cuatro estudiosos emblemáticos de las Ciencias de la Comunicación del Brasil: Luiz Beltrão, Décio Pignatari, Muniz Sodré y Sérgio Capparelli.

Esta visión general trata de ubicar exclusivamente algunas de las claves teóricas que considero como primordiales en la amplia y diversa contribución brasileña al pensamiento de la comunicación, en el contex to de la producción científica de la Escuela Latinoamericana de la Comunicación (ELACOM). El interés de este trabajo quizás estriba en subrayar algunas contribuciones sin las cuales no es posible comprender el conjunto del pensamiento brasileño de la comunicación. Parto de entender, como hipótesis de trabajo inicial, que un par de claves medulares de entendimiento en las contribuciones teóricas brasileñas están ligadas directamente con la corriente crítica y el sincretismo ético-epistemológico'. En mi opinión, ambas son trascendentales para establecer un pensamiento comunicacional propio en los estudios de la comunicación en Brasil y en América Latina en general (León, 2010: 73-190).

En este sentido, existen varios grupos de pensadores que implican desarrollos epistemológicos y éticos relativamente diferenciados. Aquí, por motivos de espacio editorial, sólo apuntamos a dos grandes grupos:

1) Un primer grupo puede ser identificado a partir de los primeros trabajos de investigación con enfoque crítico desarrollados en Brasil desde

1 Por ejemplo, con la corriente crítica en tanto cuestionan abiertamente a las dictaduras militares, la denuncia del aparato ideológico del Estado y al dominio cultural y económico de Estados Unidos de América sobre los países de América Latina y el Caribe. En mi opinión, el posicionamiento crítico de sus contribuciones generalmente tiende a ser compartido con la dimensión epistemológica y ética. Por un lado, por la diversidad teórico-metodológica que presentan bajo el denominador común de la realidad social latinoamericana. Por otro lado, por la definición ética-ideológica que tiende a posicionar las contribuciones en favor de un cambio social con justicia, igualdad y auto sustentabilidad para América Latina. Para mayor información al respecto ver: León (2008: 167-172; 2009: 135-137).

Mediaciones Sociales, № 8, I semestre 2011, pp. 3-19. ISSN electrónico: 1989-0494. DOI: $10.5209 /$ rev_MESO.2011.n8.1 
1923 y hasta la década de los años sesenta ${ }^{2}$. Se caracteriza por presentar un hibridismo metodológico y una fuerte influencia teórica de corrientes críticas y funcionalistas en el estudio de la cultura de masas ${ }^{3}$.

2) Un segundo grupo de investigadores de la comunicación surge claramente a partir de la década de los 70. La producción académica de los cuatro autores que se analizan a continuación son un ejemplo de ello. Por lo general, son estudios preocupados por la reflexión de la comunicación como un fenómeno sociocultural y multidimensional. Se puede advertir desde ya, que el factor diferencial no es tanto el método, sino el compromiso social y ético de su investigación por el cambio social en Brasil y en América Latina. Su modelo de análisis utiliza una metodología cualitativa de corte comprensivo que busca, sobre todo, la confiabilidad y el mayor grado de profundidad en el conocimiento del objeto estudiado.

\section{LUIZ BELTRÃo. FolKCOMUNiCACIÓN}

El aporte y la influencia de la obra del Profesor Luiz Beltrão de Andrade Lima ${ }^{4}$ son fundamentales para entender el pensamiento comunica-

2 Se tiene constancia de que la obra que define un pensamiento crítico de la comunicación en Brasil está cumpliendo en estas fechas 88 años. Es el trabajo pionero del pensamiento crítico que sobre temas comunicacionales llevó a cabo el investigador brasilero Barbosa Lima Sobrino. De acuerdo con Marques de Melo (2004: 16), Barbosa Lima publicó en 1923, el libro O problema da impren$s a$, donde demostró un sutil distanciamiento en relación con los procesos comunicacionales vigentes en las sociedades que disputaban la hegemonía política en el siglo XX. Asimismo, revisó críticamente las tesis defendidas por figuras emblemáticas del periodismo brasilero, como Evaristo de Vega, a la vez que repasó cuidadosamente los episodios históricos registrados por analistas del corte de Alfredo de Carvalho.

3 Nos referimos aquí a procedimientos de síntesis entre la metodología norteamericana y reflexión europea, los cuales son externos a la investigación propiamente latinoamericana. Por un lado, aquella tendencia empírica funcionalista que predominó desde 1950 hasta fines de la década de los años setenta, la Mass Communication Research. Por otro, las tendencias originarias de Europa, donde destacan principalmente la perspectiva de la teoría crítica (que a partir de 1964, con la publicación en Estados Unidos de El hombre unidimensional de Herbert Marcuse (1968: 9-274), se le empieza a nombrar e identificar en América Latina como la Escuela de Frankfurt, lo mismo que a sus destacados representantes: Adorno, Horkheimer y Benjamin) y las diferentes interpretaciones marxistas: la semiótica de Pierce y el estructuralismo francés.

4 Tanto en Brasil como en el exterior, el nombre de Luiz Beltrão de Andrade Lima (1918-1986) es sinónimo de folkcomunicación. El término folkcomunicación delimita la basta área a la cual dedicó gran parte de sus investigaciones: el conjunto de procedimientos de intercambio de informaciones, ideas, opiniones y actitudes de los públicos marginales urbanos y rurales, a través de personas y medios directa o indirectamente ligados al folklore. La tesis sobre folkcomunicación, de-

Mediaciones Sociales, № 8, I semestre 2011, pp. 3-19. ISSN electrónico: 1989-0494.

DOI: $10.5209 /$ rev_MESO.2011.n8.1 
cional del Brasil. Hoy en día, pocos estudiosos brasileños pueden dudar de que una de las principales contribuciones brasileñas a la teoría de la comunicación sea la folkcomunicación: la construcción de una teoría de la comunicación que trata de conocer y comprobar cómo la mediación de la cultura popular influye en los procesos de decodificación y las consecuentes interpretaciones en los modos de pensar, sentir y hacer que los medios de comunicación emiten cotidianamente a la población. De acuerdo con Beltrão (2001: 73), sus manifestaciones pueden ser de carácter y contenido periodístico, constituyéndose en vehículos adecuados para la promoción del cambio social. En mi opinión, la configuración del soporte teórico que desarrolla la obra de Luiz Beltrão, puede ser entendida a partir de dos textos que simbolizan la síntesis de su pensamiento.

El primero de ellos es Comunicación y folclore (Beltrão, 1971). Se trata de un estudio de los medios populares de información y expresión de las ideas, resultante de su tesis doctoral (Beltrão, 1967). En esta investigación documental y de campo, Luiz Beltrão se ocupa de trazar las directrices de la Folkcomunicación. Enfoca el estudio en el lenguaje popular, partiendo de una lengua indígena autóctona del Brasil, el tupi. Su estudio está dividido en dos partes, el lenguaje oral y la información escrita. En estas manifestaciones populares, Beltrão identifica la "folkcomunicación opinativa" que puede ser interpretada como la información, trasmutada en opinión, que es procesada a través de la sátira, de la crítica, de la caricatura y de los símbolos; son manifestaciones y rituales de las clases populares en connaturalidad entre los eventos y sus participantes (Rocha, 2000: 203).

La segunda obra de Beltrão es Folkcomunicación: la comunicación de los marginados, texto publicado en 1980 por la Editorial Cortez. Quizás el principal aporte de esta obra es la construcción de una teoría de la comunicación que trata de conocer y comprobar cómo la mediación de la cultura popular influye en los procesos de decodificación y las consecuentes interpretaciones. Es decir, con la teoría de Folkcomunicación, Luiz Beltrão comprende los procesos de intercambio de informaciones y manifestacio-

fendida en 1967, le convirtió en el primer doctor en Comunicación en Brasil. Al crear en 1997 el "Premio Luiz Beltrão de Ciencias de la Comunicación", atribuido anualmente a las personalidades o instituciones que prestaron relevantes servicios al campo de conocimiento, la Sociedad Brasileña de Estudios Interdisciplinares de la Comunicación (INTERCOM) pretendió homenajearlo como pionero de los estudios científicos de la comunicación en el Brasil. Para mayor información al respecto, ver el Portal Luiz Beltrão: http://www2.metodista.br/unesco/luizbeltrao/index.htm

Mediaciones Sociales, № 8, I semestre 2011, pp. 3-19. ISSN electrónico: 1989-0494. DOI: $10.5209 /$ rev_MESO.2011.n8.1 
nes de opiniones, actitudes e ideas del pueblo a través del folclore (Targino, 2000: 179). O más precisamente, Folkcomunicación es la comunicación a través del folclore. En palabras de Luiz Beltrão

"la Folkcomunicación es, por naturaleza, estructura, un proceso artesanal y horizontal, semejante en esencia a los tipos de comunicación interpersonal ya que sus mensajes son elaborados, codificados y transmitidos en lenguajes y canales familiares a la audiencia, por su parte conocida psicológica y vivencialmente por el comunicador, aunque dispersa” (Beltrão, 1980: 28).

El pensamiento comunicacional que expresa la producción de Luiz Beltrão, concretamente a partir de las dos obras arriba mencionadas, supone el tránsito de un sentido de la comunicación, unido inicialmente al “desarrollismo" que imprimía el Centro Internacional de Estudios Superiores de Comunicación para América Latina (CIESPAL), en las décadas del sesenta y setenta, a un modo de pensar la comunicación de masas ligado de manera íntima a las prácticas y al clima social, económico y cultural que se vivía en una determinada región y en un periodo histórico concreto del Brasil.

\section{Décio Pignatari. Contracomunicación}

Cuatro ejes temáticos pueden definir la posición teórica del profesor brasileño Décio Pignatari ${ }^{5}$ al interior del campo de la comunicación de Brasil:

1) En las cuatro décadas de existencia, la obra de estudios sobre la comunicación del profesor Pignatari se envuelve intensamente por lo que Freitas (2001: 39) llama "signagem", es decir, por los procesos sígnicos presentes en las relaciones entre el hombre y las cosas del mundo. El devenir de esta preocupación en Pignatari, es el contacto estrecho de éste con el diseño y el arte concreto.

2) Las primeras reflexiones de Pignatari sobre enseñanza de la comunicación, aparecen con su libro Contracomunicación (Pignatari, 1971a), don-

5 Décio Pignatari es doctor en Letras por la Universidad de Sao Paulo (USP), Brasil. Es profesor "Libre-Docente" de la Facultad de Arquitectura y Urbanismo de la USP. Sus libros más destacados son Información, Lenguaje y Comunicación (1971) y Contracomunicación (1971a).

Mediaciones Sociales, № 8, I semestre 2011, pp. 3-19. ISSN electrónico: 1989-0494. DOI: $10.5209 /$ rev_MESO.2011.n8.1 
de propone los principios de estructuración de una nueva escuela de comunicación. En este texto, Pignatari enfatiza la integración de medios y vehículos de comunicación, códigos y lenguajes, y la aproximación entre cuerpo docente y alumno, a la teoría de la información y la semiótica; las cuales formarían la espina dorsal teórica que subsidiaría el conocimiento de los medios de comunicación de masas. Para ello, Pignatari recurriría al investigador canadiense Marshall McLuhan para "afirmar que es preciso restituir la investigación en su sentido original de experimentación, descubriendo y creando" (Pignatari, 1971a: 23). Evidentemente, Pignatari no compartía la visión crítica Frankfurtiana, que dominó los estudios de la comunicación en Brasil desde mediados de la década de los 60 y hasta fines de los 70 .

3) Por el contrario, Pignatari representó la antítesis de ello, vislumbrando en las tesis del profesor McLuhan y el teórico estadounidense Norbert Wiener, fundador de la cibernética, como los medios idóneos y capaces de dar cuenta del nuevo espacio mediático de la comunicación, concretamente en la teoría de la comunicación y la semiótica. Por ello, es que

"en la mayoría de sus artículos es posible encontrar comentarios a favor de los mass media como instrumento cultural que en primera instancia son capaces de reunir lo que hay de mejor en todos los otros lenguajes y de hacerse presente en todas las clases sociales" (Freitas, 2001: 35).

4) Por su parte, Duarte (2001: 49-52), considera que las ideas centrales que fusionan como "matriz proliferante" el pensamiento comunicacional de Pignatari son las siguientes: a) La dialéctica sistémica de la comunicación implica contracomunicación, esto es, "sólo el incomunicable comunica: comunicación como sistema, como proceso de producción e interpretación de signos, en síntesis, capacidad de crear o diseñar lenguaje” (Duarte, 2001: 52). b) El objeto de un sistema comunicacional es su cualidad diferencial que revela el más alto grado de información de un organismo vivo. c) El diagrama del pensamiento comunicacional de Pignatari se envuelve, en principio, por una conciencia del lenguaje ya que ahí está "la tecnología de las tecnologías”. d) La capacidad simbólica de la comunicación camina de lado a lado, entre representación y comunicación; de ahí que exista una correlación inevitable entre comunicación y codificar la realidad, donde 
comunicación sería el meta-sistema de cualquier organismo vivo, que precisa comunicar para sobrevivir.

\section{Sérgio Capparelli. Enfoque multidisciplinar e investi- GACIÓN APLICADA}

Por su parte, el trabajo académico del profesor brasileño Sérgio Capparelli $^{6}$ se inscribe en el referente teórico propio de la economía política, abordando los medios de comunicación, particularmente la televisión, a partir de una inserción amplia en la estructura social, económica, política y cultural. El profesor Sérgio Caparelli promueve y desarrolla la idea de la comunicación como un área de estudio multidisciplinar. De acuerdo con el análisis de las ideas comunicacionales de Sérgio Capparelli, en la relación de los medios de comunicación con los problemas sociales, políticos y económicos emerge siempre la situación de los sectores oprimidos en la sociedad. Pero esta constatación también tiene consecuencias en otros órdenes. Por ejemplo, en el hecho de que Capparelli tiene una idea más clara de que comunicación es un área de estudio multidisciplinar, con innumerables interfaces -aunque siempre utilice el paradigma de la economía política-, donde se percibe una profundidad de enfoques y de perspectivas teóricometodológicas, que actualmente circulan en la comunicación (Chitto, 2001: 117). Los ejes temáticos que definen la posición del pensar, decir y hacer de Capparelli en materia de teoría de la comunicación en Brasil son:

1) Desde que surge el trabajo de Sérgio Capparelli, su propuesta ha sido realizar investigaciones en comunicación con un carácter concreto, significando con esto, iniciar la investigación con un diagnóstico de comunicación, a partir de la realidad y el uso racional de la comunicación en los procesos que se vivencian en América Latina. Es decir, para Capparelli la investigación en comunicación podía tornarse en un medio instrumental para mejorar el conocimiento de Brasil y de América Latina.

\footnotetext{
6 Sérgio Capparelli es profesor de la Universidad Federal de Río Grande del Sur, Brasil. Entre su producción sobre estudios de comunicación destacan: Comunicación de Masas sin Masas (1979), Televisión y Capitalismo en Brasil (1982), Dictadura e Industrias culturales en Brasil, Argentina, Chile y Paraguay (1989) y Al Fin Solos: La Nueva Televisión en el Cono Sur (Albornoz y Capparelli, 1999).
}

Mediaciones Sociales, № 8, I semestre 2011, pp. 3-19. ISSN electrónico: 1989-0494. DOI: $10.5209 /$ rev_MESO.2011.n8.1 
2) Ello se muestra claramente en su primer libro, Comunicación de masas sin masas (1979), donde, a partir de detectar que si bien las empresas periodísticas de Brasil contaban con las condiciones tecnológicas que hacían posible la producción masiva de periódicos, concluyó que el consumo fue siempre impedido por las condiciones de analfabetismo y de la situación económica de la población. Esta falta de masas, para el autor, da lugar a que la radio y la televisión se instalasen con tanta intensidad, principalmente en Brasil (Chitto, 2001: 111).

3) La distinción epistemológica que define el abordaje teóricometodológico de la comunicación de la propuesta de Capparelli es su articulación con un fondo analítico amplio, que comprende a la economía, la política, la cultura y a la sociedad; en Televisión y capitalismo en Brasil (Capparelli, 1982), detalla dicho abordaje a partir situar a la televisión brasileña dentro del modelo capitalista de producción. Capparelli concretaría con claridad la implantación de la televisión en Brasil: inicia su estudio desde 1964, para criticar y denunciar cómo este medio de comunicación (al igual que lo haría con la radio y el periódico), es una de las causas del capitalismo dependiente impuesto en la sociedad brasileña, porque, según Capparelli,

“además de divertir o instruir, el medio asume funciones ligadas al sistema capitalista: ofrece nuevas posibilidades al capital; propaganda de productos; ampliación del mercado y del sector comunicación; por llevar la idea de necesidad de consumo para generaciones de la sociedad que anteriormente no participaban del mercado y la difusión y refuerzo de ideología dominante" (Capparelli, 1982. Citado en Chitto, 2001: 111).

4) De manera específica, uno de los primeros aportes de la obra de Capparelli a los estudios de la comunicación en Latinoamérica se concretiza al finalizar la década de los 80, con su publicación Dictadura e industrias culturales en Brasil, Argentina, Chile y Paraguay (Capparelli, 1989).

En esta obra, de nueva cuenta, Capparelli abordará la comunicación, analizando la radio, la televisión y la imprenta, en relación con los aspectos políticos, económicos y sociales. La vertiente política de la obra desvela el uso con el que fue hecho desde los medios, el alcance y los objetivos políticos del poder de Estado. Respecto a la relación con la situación económica, Chitto apunta que esta esfera muestra que el modelo de industrialización dependiente no fue exclusiva de países del cono sur, mas aquí encontró el terreno fértil para aliarse a los grupos dominantes del capital internacional 
"con relación a la situación social, el análisis proviene de la historia de estos pueblos permeada por la situación política y económica a la que fueron sometidos durante las dictaduras que se implantaron casi simultáneamente en los países del cono sur (...) el autor ya incluye un pasaje de esos países para la democracia, mostrando cómo los medios de comunicación contribuirían para que esto ocurriera" (Chitto, 2001: 114-115).

Para Ramos (2001: 119-124), el aporte de la obra de Capparelli a los estudios de la comunicación en Latinoamérica se centra en dos aspectos significativos: 1) En el estudio de los efectos de las instituciones regionales de comunicación, especialmente las que proyectan políticas neoliberales o políticas económicas heredadas del neoliberalismo "thatcherista" y "reganiano". 2) En la década de los 90, Capparelli amplía su foco de análisis al escenario de la comunicación en el cono sur. El estudio principal y su aporte se centran en la televisión. De aquí nace el proyecto de investigación sobre la televisión en el cono sur que durante tres años, y bajo el liderazgo del profesor Capparelli, llevaron a cabo investigadores de las universidades de Buenos Aires, la Católica de Montevideo, la Federal Río Grande del Sur y la Universidad de Brasilia. Los resultados de la investigación se publicaron en: $A l$ fin solos: la nueva televisión en el cono sur (Albornoz y Capparelli, 1999).

Por otro lado, queda patente la posición de Capparelli (2001: 94-95) de relacionar y conectar productivamente el trabajo científico con la actividad política. Acredita que la biografía de un investigador, su visión del mundo, la realidad política de su país, las variaciones culturales con las que convive, la estructura universitaria en las que participa, etc., son elementos que forman parte e inciden de manera muy fuerte en la investigación dentro del campo de la comunicación. De ahí que, desde su opinión, se pueda aceptar la realidad tal como está y cooperar para que ésta avance en una u otra dirección; considera que las perspectivas de análisis de la comunicación no avanzan cronológicamente sino que unas son sustituidas por otras; para el investigador brasileño, tanto los temas como los abordajes en la investigación de la comunicación son recurrentes y son sustituidos y ultrapasados debido a la dinámica propia de la realidad; se renuevan y son vueltos a situar en el centro de interés?

7 Como ejemplo de ello, Capparelli ubica los estudios sobre la economía política crítica en el actual escenario de globalización y de tecnologías digitales. Argumenta que en esta corriente de estudio los instrumentos de análisis no son inmutables pues "pasan por un refinamiento al

Mediaciones Sociales, № 8, I semestre 2011, pp. 3-19. ISSN electrónico: 1989-0494. DOI: $10.5209 /$ rev_MESO.2011.n8.1 
Queda claro que para el profesor Capparelli (2001: 91), el trabajo de investigación científica debe entenderse como una vía de múltiples acciones que éste tiene por recorrer, como presupuestos teóricos que hace uso en tanto son procedimientos y opciones en su vida científica. En este sentido, procura situar históricamente algunas de sus opciones por temas y abordajes, construyendo un diálogo con su memoria histórica personal. Por ello, no acepta que el investigador en comunicación sea inocente. Tiene y traza sus valores, sus principios, su manera de ver el mundo, sus prioridades dentro de la vida social. Al mismo tiempo, encuentra objetos de estudio que tienen su propia lógica, sus ingredientes y su trayectoria dentro de la sociedad. También, para Capparelli (2001: 92-93), las opciones de investigación significan pérdidas. Pérdidas del objeto, debido a la opción hecha y debido al recorte hecho. También él tiene iluminaciones, a manera de los artistas, y las entiende más como un producto de síntesis que de análisis. Mucho más de creación que de racionalidad. Es justamente en dichas iluminaciones, donde Capparelli ubica la relación y la proximidad entre investigador y poeta, en tanto tienen procesos de estados febriles de creación, los cuales casi siempre acontecen en momentos inesperados: en la hora del almuerzo, al ir caminando rumbo a la escuela, en medio de un filme, entre otros.

\section{Muniz Sodré. Comportamiento Indisciplinar de la Co- MUNICACIÓN}

La obra de estudio de la comunicación del investigador brasileño $\mathrm{Mu}$ niz Sodré 8 , puede ubicarse dentro de una multiplicidad de temáticas y su proposición básica es la lectura crítica de los medios. Para Paiva, por ejemplo, la obra de Muniz Sodré se relaciona siempre con la lectura crítica de la producción mediática de la sociedad, el contexto político, económico e histórico y, justamente por esa razón, es que su investigación hace uso de

comenzar a hacer empleados con una mayor flexibilidad y, principalmente, bajo una conciencia de ser apenas una de las puertas explicativas para los fenómenos comunicativos" (Cappare1li, 2001:95).

8 Muniz Sodré es profesor y coordinador del Postgrado en Comunicación en la Universidad Federal de Río Grande del Sur, Brasil. Entre su obra reciente se destaca: Reinventando la Cultura: la Comunicación y sus Productos (1998) y Sociedad, Cultura y Violencia (2001).

Mediaciones Sociales, № 8, I semestre 2011, pp. 3-19. ISSN electrónico: 1989-0494. DOI: $10.5209 /$ rev_MESO.2011.n8.1 
varios saberes y campos de conocimiento, no privilegiando un campo teórico determinado o una metodología específica (Paiva, 2001: 83).

La especificidad que marca uno de los pilares de estudio en Sodré viene marcada por el abordaje de los productos generados por la cultura de masas, el estudio de la cultura y la identidad nacional: "Un ejemplo de eso es su análisis de medios brasileños, cuyo resultado, casi una profecía, fue el libro La comunicación de lo grotesco, un ensayo sobre cultura de masas en Brasil, publicado en 1972 por la editora Vozes" (Paiva, 2001: 82).

No obstante lo anterior, para el propio Sodré (2001a: 71-75), si existe actualmente un campo de conocimiento con una posición reflexiva sobre la vida social y con un objeto claro, es precisamente la comunicación. Para este investigador, el comportamiento "indisciplinar" de los estudios de la comunicación resulta de la incómoda condición de estar comprimida entre las grandes disciplinas de pensamiento social y de una multiplicidad de prácticas socioculturales activas, que generalmente lanzan una sombra sobre el objeto. Sin embargo, su posición es franca: "la comunicación tiene como objeto la vinculación entre el yo y el otro, sea considerando el punto de vista individual sea el colectivo" (Sodré, 2001a: 71-75).

De ahí que Sodré ubique a la comunicación desde varios modos y niveles de estudio como ha pasado en la historia de los sistemas de pensamiento, desde Platón y Aristóteles, pasando por Husserl, Heidegger o Habermas. Según Sodré, la comunicación ha sido tratada, primero, como un subtema de disciplinas del pensamiento social sistematizado en el siglo XIX y, ahora, se encamina progresivamente para una posición de autonomía relativa en faz de las disciplinas sociales y humanas ya consolidadas. Con todo, la concepción más novedosa que Sodré (2001a: 72-74), aporta al campo de estudios de la comunicación se relaciona directamente con la autonomía relativa que tiene el objeto de estudio de la comunicación. En este sentido, Sodré afirma que la garantía que tiene la comunicación respecto a su autonomía en el objeto de estudio, viene dada por su especificidad de vinculación social que, en sentido lato, es el objeto de estudio de una ciencia de la comunicación. En sentido estricto, la evidencia de que las prácticas socioculturales, comunicacionales o mediáticas vienen instituyéndose como un campo de acción social correspondiente a una nueva forma de vida, que propone llamar "bios-mediático" (Sodré, 2001a: 72-74). 
El "bios-mediático" es, para Sodré (2001a: 74), la resultante de la evolución de los medios y de su progresiva intersección con las formas de vida tradicionales. Indica que en una ciencia de la comunicación cabrá la producción de conocimiento específico (y no marcadamente sociológico, antropológico, psicológico, periodístico, etc.) sobre la socialización recurrente de esa nueva realidad histórica que, en todo caso, indica Sodré, requerirá de un nuevo sistema de pensamiento basado en una anarquía de ideas y teorías tal y como lo propondría Feyerabend (1993) en Contra el método.

\section{ConClusiones}

Con el desarrollo epistemológico y la aplicación concreta de la investigación en comunicación elaborada por los cuatro científicos brasileños aquí analizados, podemos concluir, siguiendo nuestra hipótesis de trabajo inicial, que sus contribuciones definitivamente están ligadas directamente con la corriente crítica y el sincretismo ético-epistemológico. En primer término, porque en todos y cada uno de los casos analizados, sus temas y objetos de estudio superan, por un lado, los límites de interacción entre varias disciplinas sociales tradicionalmente cerradas y jerárquicas, como campo de conocimiento específico. También, porque sus contribuciones asumen que el estudio de la comunicación no sólo representa un detonador simbólico notable en las transformaciones económicas, políticas, ideológicas y culturales de la sociedad brasileña contemporánea, sino en el ofrecimiento de evidencia empírica dura que demuestra que el campo de la comunicación tiene un objeto de estudio eminentemente sociocultural, multidimensional y centrado en seres humanos y procesos de producción de sentido.

Efectivamente, ha quedado asentado en cada una de las cuatro propuestas teóricas analizadas, la iniciativa de patentizar el estudio de la comunicación como un proceso social de entendimiento, que enfatiza las condiciones dinámicas y de cambio social. Al igual y como sucede con no pocas obras e historias académico-científicas en Latinoamérica (León, 2010: 129-151), el colofón identitario de los teóricos brasileños aquí analizados parece no ser otro que la plataforma ético-política de la comunicación, amalgamada con una visión crítica y socio-histórica de sus temas, objetos y métodos de estudio. 


\section{BibLIOGRAFÍA}

Albornoz, Luis y CAPARELli, Sérgio (1999): Al fin solos: la nueva televisión en el cono sur. Buenos Aires: Ciccus-La Crujía.

BELtRÃo, Luiz (1967): Folkcomunicação: um estudo dos agentes e dos meios populares de informação de fatos e expressão de idéias. Tese de Doutoramento. Brasília: UnB.

BELtrão, Luiz (1971): Comunicação e folclore. São Paulo: Melhoramentos.

BELTRÃo, Luiz (1980): Folkcomunicação, a comunicação dos marginalizados. São Paulo: Cortez.

BELTRÃo, Luiz (2001): Folkcomunicação: um estudo dos agentes e dos meios populares de informação de fatos e expressão de idéias. Porto Alegre: EDIPUCRS.

Beltrão, Luiz (2001a): Folkcomunicação: teoria e metodología. São Bernardo do Campo: Umesp.

Capparelli, Sérgio (1979): Comunicación de masas sin masas. São Paulo: Cortez.

CAPPARElli, Sérgio (1982): Televisão e capitalismo no Brasil. Porto Alegre: LPM.

Capparelli, Sérgio (1989): Ditadura e indústrias culturais no Brasil, na Argentina, no Chile e no Paraguay. Porto Alegre: Editora da Universidade/UFRGS.

CAPParelli, Sérgio (1998): “Cenário e espelhos”, en Marques De Melo, José y GoRsKI, Juçara: A trajetória comunicacional de Luiz Ramiro Beltrán. São Paulo: UNESCO, pp. 53-56.

CAPparelli, Sérgio (2001): "Economia política da paixão pela pesquisa", en Marques de Melo, José, GobBi, Maria Cristina y Dos SAntos, Marli: Contribuições brasileiras ao pensamento comunicacional latino-americano: Décio Pignatari Muniz Sodré e Sérgio Capparelli. São Paulo: UNESCO, pp. 91-102.

CHITO, Ida (2001): “Idéias comunicacionais de Sérgio Capparelli”, en MARQUES DE Melo, José, GobBI, Maria Cristina y Dos SAntos, Marli: Contribuições brasileiras ao pensamento comunicacional latino-americano: Décio Pignatari Muniz Sodré e Sérgio Capparelli. São Paulo: UNESCO, pp. 109-118.

Duarte de Oliveira, Maria (2001): “Décio Pignatari: o choque do novo", en Marques de Melo, José, Gobbi, Maria Cristina y Dos Santos, Marli: Contribuições brasileiras ao pensamento comunicacional latino-americano: Décio Pignatari Muniz Sodré e Sérgio Capparelli. São Paulo: UNESCO, pp. 109-118. 
Feyerabend, Paul (1993): Contra el método. Barcelona: Planeta.

Freitas, Hélio (2001): "Décio Pignatari: designer da linguagem”, en MarQues DE Melo, José, GobBI, Maria Cristina y Dos Santos, Marli: Contribuições brasileiras ao pensamento comunicacional latino-americano: Décio Pignatari Muniz Sodré e Sérgio Capparelli. São Paulo: UNESCO, pp. 35-48.

LEÓn, Gustavo (2008): Escola Latino-Americana de Comunicacão. A nova hegemonia. São Paulo: Cátedra UNESCO Comunicação Brasil-UMESP.

LEÓn, Gustavo (2009): La nueva hegemonía en el Pensamiento Latinoamericano de la Comunicación. México: PEARSON-Prentice Hall.

LEÓn, Gustavo (2010): Sobre la institucionalización de la comunicación en América Latina. México: PEARSON-Prentice Hall.

MARCUSE, Herbert (1968): El hombre unidimensional. Ensayo sobre la ideología de la sociedad industrial avanzada. México: Joaquín Mortiz.

Marques De Melo, José (1978): Comunicação, modernização e difusão de inovações no Brasil. Petrópolis: Vozes.

Marques de Melo, José (1979): "Ensino de comunicação no Brasil”, en MARQUES DE MELO, José, FADUL, Anamaria y Lins, Carlos: Ideología y poder no ensino de comunicação. São Paulo: Cortes y Moraes, pp. 31-41.

Marques de Melo, José (1983): Teoria e pesquisa em comunicação. Panorama latino-americano. São Paulo: INTERCOM/Cortez.

Marques de Melo, José (2001): "Pensamento comunicacional brasileiro no cenário da Escola Latino-Americana de Comunicação”, en MARQues DE MELO, José, GobBI, Maria Cristina y Dos SAnTos, Marli: Contribuições brasileiras ao pensamento comunicacional latino-americano: Décio Pignatari Muniz Sodré e Sérgio Capparelli. São Paulo: UNESCO, pp. 23-34.

MARques de Melo, José (2004): “Prefacio”, en MARQues de Melo, José y GobBI, Maria Cristina: Pensamento comunicacional latino-americano. São Paulo: UNESCO, pp. 15-18.

Marques de Melo, José (2004a): "Los tiempos heroicos. La formación de la comunidad latinoamericana de la comunicación”, Revista Latinoamericana de Ciencias de la Comunicación, $\mathrm{n}^{\mathrm{o}}$ 1, pp. 10-21.

Mediaciones Sociales, № 8, I semestre 2011, pp. 3-19. ISSN electrónico: 1989-0494.

DOI: $10.5209 /$ rev_MESO.2011.n8.1 
«Luiz Beltrão, Décio Pignatari, Muniz Sodré y Sérgio Capparelli. Aportes teóricos...»

Marques De Melo, José (2004b): A esfinge mediática. São Paulo: Paulus.

Marques De Melo, José (2005): Midiologia para iniciantes. Uma viagem coloquial ao planeta mídia. Caxias do Sul, Brasil: EDUCS.

Marques de Melo, José, Gobbi, Maria Cristina y Dos Santos, Marli (2001): Contribuições brasileiras ao pensamento comunicacional latino-americano: Décio Pignatari Muniz Sodré e Sérgio Capparelli. São Paulo: UNESCO.

PAIVA, Raquel (2001): "Pluralidade cultural”, en MARQues DE Melo, José, GobBI, Maria Cristina y Dos SANTOS, Marli: Contribuições brasileiras ao pensamento comunicacional latinoamericano: Décio Pignatari Muniz Sodré e Sérgio Capparelli. São Paulo: UNESCO, pp. 81-84.

PIGNATARI, Décio (1971): Información, lenguaje y comunicación. Quito: CIESPAL.

Pignatari, Décio (1971a): Contracomunicación. São Paulo: Paulus.

Ramos, César Murilo (2001): "Sérgio Capparelli: a centralidade de uma trilogía", en Marques de Melo, José, GobBI, Maria Cristina y Dos Santos, Marli: Contribuições brasileiras ao pensamento comunicacional latino-americano: Décio Pignatari Muniz Sodré e Sérgio Capparelli. São Paulo: UNESCO, pp. 119-124.

RoCHA, Samantha (2000): "Luiz Beltrão: da criação do ICINFORM à teoria da folkcomunicação”, en MARQues De Melo, José y GobBI, Maria Cristina: Gênese do pensamento comunicacional latino-americano: o protagonismo das instituições pioneirasCIESPAL, ICINFORM, ININCO. São Paulo: UNESCO, pp. 193-212.

SODRÉ, Muniz (1998): Reinventando la cultura: la comunicación y sus productos. Barcelona: Gedisa.

SoDRÉ, Muniz (2001): Sociedad, cultura y violencia. Bogotá: Norma.

SODRÉ, Muniz (2001a): "Bios mediático: um novo sistema conceptual no campo da comunicação”, en MARQues DE Melo, José, GobBI, Maria Cristina y Dos SANTOS, Marli: Contribuições brasileiras ao pensamento comunicacional latino-americano: Décio Pignatari Muniz Sodré e Sérgio Capparelli. São Paulo: UNESCO, pp. 71-76.

TARGINO, Maria (2000): “A contribuição do ICINFORM na gênese do pensamento comunicacional brasileiro”, en MARQUES DE MELo, José y GOBBI, Maria Cristina: Gênese do pensamento comunicacional latino-americano: o protagonismo das instituições pioneiras- CIESPAL, ICINFORM, ININCO. São Paulo: UNESCO, pp. 167-180. 


\section{PARA CITAR ESTE TRABAJO EN BIBLIOGRAFÍAS:}

LEÓN DUARTE, Gustavo A. (2011): “Luiz Beltrão, Décio Pignatari, Muniz Sodré y Sérgio Capparelli. Aportes teóricos brasileños al pensamiento latinoamericano de la comunicación", Mediaciones Sociales. Revista de Ciencias Sociales y de la Comunicación, $\mathrm{n}^{\mathrm{o}}$ 8, pp. 3-19. DOI: 10.5209/rev_MESO.2011.n8.1; http://dx.doi.org/10.5209/rev MESO.2011.n8.1

\section{${ }^{(*)}$ El autor}

Gustavo A. León Duarte es líder del Grupo Enseñanza e Investigación de la Comunicación en América Latina (GEIC-AL). Es profesor investigador titular en el Departamento de Psicología y Ciencias de la Comunicación de la Universidad de Sonora (México). Es licenciado en Ciencias de la Comunicación por la Universidad de Sonora y master y doctor en Periodismo y Ciencias de la Comunicación por la Universidad Autónoma de Barcelona (España). Es miembro del Sistema Nacional de Investigadores de México (Consejo Nacional de Ciencia y Tecnología de México - Conacyt) y evaluador acreditado del Consejo Nacional de Ciencia y Tecnología de México (RCEA), en el área de las Ciencias Sociales y Económicas. Ha impartido cátedra y conferencias en pre y postgrado en varias universidades de EE.UU. e Hispanoamérica. Ha publicado más de 60 artículos en libros y revistas especializadas y es autor de varias obras como La nueva hegemonía en el Pensamiento Latinoamericano de la Comunicación (2009, $2^{\mathrm{a}}$ ed.), Sobre la institucionalización de la comunicación en América Latina (2010), Estudios de la comunicación. Estrategias metodológicas y competencias profesionales en comunicación (2011). Correo electrónico: gustavoadolfoleon@gmail.com

RECIBIDO: 22 de febrero de 2011.

ACEPTADO: 9 de junio de 2011. 
